\title{
Editorial
}

\section{La investigación en enfermería: un constante reto para la disciplina}

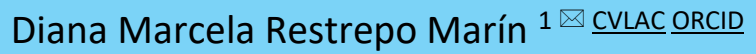

${ }^{1}$ Enfermera, Magister Epidemiología, Magister Control de Infecciones y Epidemiología Hospitalaria, Docente, Universidad CES.

Fecha correspondencia:

Recibido: diciembre 15 de 2021.

Aceptado: diciembre 15 de 2021.

Forma de citar:

Restrepo-Marín DM. La

investigación en enfermería: un constante reto para la disciplina. Rev CES Enf [Internet]; 2(2):1-4. Disponible en: https://dx.doi.org/ $\underline{10.21615 / \text { cesenferm.6545 }}$

$\underline{\text { Open access }}$

Licencia creative commons

Ética de publicaciones

Revisión por pares

Gestión por Open Journal System DOI: 10.21615/cesenferm.6545

ISSNe 2745-049X

Publica con nosotros
Según Alarcón, la investigación constituye un factor determinante para el desarrollo de una ciencia (1); aunque la ciencia de enfermería ha sido objeto de investigación rigurosa, hay algunas desigualdades importantes en cuanto a la capacidad de los enfermeros para investigar y aplicar los resultados de las investigaciones en su práctica diaria. Pese a que el número de investigaciones y publicaciones en enfermería ha aumentado en la última década, la disciplina se enfrenta a grandes y constantes retos $^{(2)}$.

Algunos autores describen como las principales barreras que enfrentan los enfermeros para desarrollar y aplicar la investigación en la práctica de enfermería, las siguientes: falta de educación en metodología de la investigación, falta de habilidades en una segunda lengua, falta efectiva de tiempo, problemas para acceder a publicar en revistas de alto nivel, baja visibilidad de la propiedad intelectual, falta de acceso a revistas científicas de enfermería, percepción de complejidad del área de investigación, bajo nivel de interdisciplinariedad, falta de educación de posgrado, falta de una agenda de investigación de enfermería para la región, carencia de incentivos en las políticas de investigación y falta de financiamiento para la investigación ${ }^{(3-5)}$.

Pese a lo anterior, múltiples estrategias se abordan en la actualidad para intervenir dichas barreras en investigación y 
para cambiar la percepción que tienen los enfermeros acerca del uso de la producción científica en el desarrollo de la disciplina, algunas de estas estrategias son implementadas desde los entornos académicos, políticos y gremiales. Por ejemplo, en la formación profesional de pregrado y posgrado, los programas académicos se han visto fortalecidos y promueven el uso y desarrollo de la investigación en los estudiantes, a su vez en el ámbito político y gremial se han generado políticas específicas que promueven el desarrollo y liderazgo de la profesión ${ }^{(6)}$.

Estos esfuerzos serán en vano si las investigaciones desarrolladas por los enfermeros no aportan al conocimiento y discusión reflexiva de los problemas que realmente impactan a la sociedad, por esto los centros de investigación deben estar orientando las líneas de investigación a los contextos actuales, basándose en las prioridades de investigación de las regiones, reconociendo que las políticas de salud y las fuentes de financiamiento ejercen una poderosa influencia sobre el proceso de generar investigaciones congruentes con la contingencia social y demográfica ${ }^{(1)}$.

Para impulsar la investigación en Enfermería, los profesionales colombianos deben enfocar sus investigaciones en temas que den solución a los problemas de su ejercicio profesional en las diferentes áreas, por lo tanto, se deben conocer las prioridades locales, nacionales e internacionales en investigación y los problemas prioritarios propios de la disciplina, de lo contrario, las publicaciones científicas no serán aplicables a la práctica, sin desconocer que este conocimiento es un desafío ${ }^{(7)}$.

En referencia a lo anterior, Hopia y col realizaron una investigación sobre las prioridades en investigación en enfermería a nivel mundial y advierten que es imperativo desarrollar investigaciones en temas como: el desarrollo de la teoría de enfermería, en particular, las teorías de rango medio y específicamente sobre situaciones que vinculan la teoría con la práctica, adicionalmente mencionan como prioritarios temas como la metodología de la investigación en enfermería, la practica avanzada en enfermería y la práctica profesional de la enfermería que involucra diversos temas clínicos como la oncología, la pediatría, entre $\operatorname{otros}^{(7-8)}$.

Otros autores plantean temas a priorizar en la región como son: políticas y educación de recursos humanos en enfermería, organización y dinámica de los sistemas y servicios de salud; ciencia, tecnología, innovación y sistemas de información en salud, políticas de salud, gobernanza y control social, estudios sociales en el ámbito de la salud, atención a la madre, atención al paciente con enfermedades crónicas no trasmisibles, atención al paciente 
oncológico, atención al niño y neonato en estado crítico de salud, práctica avanzada en enfermería, seguridad del paciente, envejecimiento en la población, imagen social del profesional de enfermería ${ }^{(2,8)}$.

Son muchos los temas en los que se debe profundizar, la invitación y el reto al cual nos enfrentamos como disciplina es a generar conocimiento relevante que aporte al fortalecimiento del quehacer enfermero, a generar espacios de difusión de dicho conocimiento y a llevarlo a la práctica profesional. Hoy más que nunca, los profesionales de enfermería están preparados para asumir este reto.

\section{Referencias}

1. Alarcon A, Astudillo P. Nursing research in Latin American Journals. Cienc. enferm. 2007: 13(2): 25-31. [citado 2021 Diciembre 15]. Disponible en: http://dx.doi.org/10.4067/S0717-95532007000200004.

2. Garcia A, Cassiani S, Reveiz L. A systematic review of nursing research priorities on health system and services in the Americas. Rev Panam Salud Publica [Rev Panam Salud Publica. 2015;37(3):162-71. [citado 2021 Diciembre 15] Disponible en: http://www.scielosp.org/scielo.php?script=sci arttext\&pid=S1020$49892015000300006 \& \operatorname{lng}=e n$

3. González A, Díez A, Martín N, Pozuelo D, Mirón R, Solera M. Barriers and Facilitators Perceived by Spanish Experts Concerning Nursing Research: A Delphi Study. Int. J. Environ. Res. 2020; 17(9):3224. [citado 2021 Diciembre 15] Disponible en: https://doi.org/10.3390/ijerph17093224.

4. Vélez C, Pico ME, Ariza C, Castellanos F, Salazar AM. Tendencias y agenda de prioridades de investigación en unidades académicas de educación superior de Enfermería en Colombia. Cienc. enferm. 2014; 20( 3 ): 11-20. [citado 2021 Diciembre 15] Disponible en: http://dx.doi.org/10.4067/S0717-95532014000300002.

5. Parmar J, House J, Cassiani S, Reveiz L. Health literature authored by nurses within the LAC region: a cross-sectional study. Rev Panam Salud Publica. 2015; 37(6):409-414 [consultado 2021 Diciembre 15] Disponible en: http://www.ncbi.nlm.nih.gov/pubmed/26245176 
6. CTE, ACOFAEN, ANEC, OCE, TNEe, ACOEEN, Ministerio de Salud y Protección Social. Política nacional de enfermería y plan estratégico 2020- 2030 Resignificando la profesión de Enfermería en Colombia. [Internet] [consultado 2021 Diciembre 15] Disponible en: https://www.anec.org.co/images/plan nacional enfermeria.pdf

7. Hopia, H, Heikkilä, J. Nursing research priorities based on CINAHL database: A scoping review. Nursing Open. 2020; 7: 483- 494. [consultado 2021 Diciembre 15] Disponible en: https://aplicacionesbiblioteca.udea.edu.co:2399/10.1002/nop2.428

8. Cassiani S, Bassalobre-Garcia A, Reveiz L. Universal Access to Health and Universal Health Coverage: identification of nursing research priorities in Latin America. Revista LatinoAmericana de Enfermagem. 2015; 23(6): 1195-1208. [Accedido 15 Diciembre 2021]. Disponible en: https://doi.org/10.1590/0104-1169.1075.2667 